\title{
Expression of key enzymes in the mevalonate pathway are altered in monocrotaline-induced pulmonary arterial hypertension in rats
}

\author{
DONGMEI JIANG ${ }^{1 *}$, YU CHEN $^{2 *}$, YUXIANG ZHU ${ }^{2}$, GUOSHENG FU ${ }^{1}$ and SHIMING XU $\mathrm{XU}^{1,2}$ \\ ${ }^{1}$ Department of Cardiology, Sir Run Run Shaw Hospital, School of Medicine, Zhejiang University, Hangzhou, Zhejiang 310016; \\ ${ }^{2}$ Institute of Translational Medicine, School of Medicine, Zhejiang University, Hangzhou, Zhejiang 310029, P.R. China
}

Received January 7, 2017; Accepted August 31, 2017

DOI: $10.3892 / \mathrm{mmr} .2017 .7798$

\begin{abstract}
Pulmonary arterial hypertension (PAH) is a serious pulmonary vascular disease. The changes in the structure, function and metabolism of endothelial cells are some of the important features of PAH. Previous studies have demonstrated that the mevalonate pathway is important in cardiovascular remodeling. However, whether the mevalonate pathway is involved in the development of PAH remains to be elucidated. The present study aimed to investigate the expression pattern of mevalonate pathway-related enzymes in monocrotaline (MCT)-induced PAH. F344 rats were randomly divided into two groups ( $n=6 /$ group): Control group rats were injected with a single dose of saline, and MCT group rats were injected with a single dose of MCT $(60 \mathrm{mg} / \mathrm{kg})$. After 4 weeks, the right ventricular systolic pressure (RVSP) was measured, and lung and pulmonary artery tissue samples were collected. It was demonstrated that the RVSP increased and pulmonary vascular remodeling was detected in the $\mathrm{PAH}$ group. The expression levels of the enzymes farnesyldiphosphate synthase farnesyltransferase $\alpha$ and geranylgeranyltransferase type I increased in the PAH group, which suggested that the mevalonate pathway may be involved in the pathological development of PAH.
\end{abstract}

Correspondence to: Dr Guosheng Fu, Department of Cardiology, Sir Run Run Shaw Hospital, School of Medicine, Zhejiang University, 3 East Qingchun Road, Hangzhou, Zhejiang 310016, P.R. China

E-mail: fu_guosheng@163.com

Mr. Shiming Xu, Institute of Translational Medicine, School of Medicine, Zhejiang University, 268 Kaixuan Road, Hangzhou, Zhejiang 310029, P.R. China

E-mail: shimingxu@zju.edu.cn

*Contributed equally

Key words: mevalonate pathway, pulmonary arterial hypertension, farnesyldiphosphate synthase, farnesyltransferase $\alpha$, geranylgeranyltransferase type I, small G-protein

\section{Introduction}

Pulmonary arterial hypertension (PAH) is a serious pulmonary vascular disease, which is defined by a mean pulmonary arterial pressure $\geq 25 \mathrm{mmHg}$ (1). The pathological features of PAH include distal pulmonary artery intimal hyperplasia, plexiform lesions, medial hypertrophy, muscle infarction and thrombosis, gradually leading to occlusion of the lumen and high pulmonary arterial pressure, eventually leading to right heart failure $(2,3)$. In previous years, several studies have demonstrated that the structure, function and metabolic changes of endothelial cells are important features in the development of $\mathrm{PAH}$, which has a marked effect on vessel homeostasis (4-6).

A typical feature of endothelial injury is an imbalance of nitric oxide (NO)-reactive oxygen species (ROS) levels, which is caused by reductions in endothelium-derived NO synthesis, release and activity, and is increased by ROS generation and release $(7,8)$, which is involved in the excessive contraction and remodeling of pulmonary vessels. It has been demonstrated that the small G proteins, Ras homolog family, member A (RhoA) and Ras-related C3 botulinum toxin substrate 1 (Rac1), are involved in changes of endothelial function through the Rho kinase-endothelial NO synthase (eNOS) pathway or regular NADPH oxidase, respectively (9). Therefore, repairing endothelial function and reversing excessive oxidative stress represent he pathophysiological foundation of PAH therapy (7).

Previous studies have demonstrated that the mevalonate pathway is involved in small G-protein activation $(10,11)$. The mevalonate pathway is an important pathway of cholesterol synthesis at the cellular level. Mevalonate is a precursor of cholesterol, in addition to several non-steroid isoprenoid complexes, including farnesylpyrophosphate (FPP) and geranylgeranylpyrophosphate (GGPP) (12). These non-steroid isoprenoids are important in small $\mathrm{G}$ protein post-translational modification (10). Studies have indirectly demonstrated that the mevalonate pathway maybe involved in the development of PAH. It has been suggested that mevalonate and other mid products produced in the mevalonate pathway can regulate signaling proteins, including those of Ras family (13), which are essential for cell proliferation and other important features. The inhibitor of the 3-hydroxy-3-mehtylglutaryl-coenzyme A (HMGR) enzyme, a key enzyme of the mevalonate pathway, decreases blood lipids, inhibits the proliferation of smooth muscle cells, ameliorates endothelial cell function and 
increases the expression of eNOS $(14,15)$. Animal experiments have shown that statins were able to mitigate pulmonary pressure and right ventricular hypertrophy in monocrotaline (MCT)-induced PAH rats, and this was associated with an increased expression of eNOS $(16,17)$.

At present, the effects of key enzymes, including farnesyldiphosphate synthase (FDPS), farnesyltransferase $\alpha$ (FNTA), farnesyltransferase $\beta$ (FNTB) and geranylgeranyltransferase type I (GGTase-I) on endothelial dysfunction have not been reported, with the exception of the initial enzyme, HMGR. The pathway downstream of FPP produced in the mevalonate pathway has three branches: Asterol branch, which primarily contributes to cholesterol synthesis, and two nonsterol branches, which regulate signal transduction proteins, including those of the Ras and Rho families $(13,14)$. Therefore, the mevalonate pathway may affect protein prenylation, vasoactive substance generation and endothelial function, and regulate excessive vascular contraction and remodeling in the pulmonary vascular tissues of PAH rats.

Based on previous studies, the present study aimed to determine whether the expression of key enzymes, including HMGR, FDPS, FNTA, FNTB and GGTase-I, in the mevalonate pathway are altered in MCT-induced PAH rats, which may potentially serve as novel therapeutic targets for PAH.

\section{Materials and methods}

Animal model. The present study was performed in adherence with the National Institutes of Health Guidelines for the Care and Use of Laboratory Animals andapproved by the ethics committee of Zhejiang University (approval no. ZJU20170874; Hangzhou, China). A total of 100 F344 male rats (6-weeks old; weighing $200 \pm 10 \mathrm{~g}$ ) were obtained from Shanghai Laboratory Animal Center (Chinese Academy of Sciences, Shanghai, China). A total of 5 rats/cage, temperature of $22^{\circ} \mathrm{C}$, light and dark cycle 12-h, free access to drinking water and normal feed. The rat PAH model was induced by injecting a single dose of MCT $(60 \mathrm{mg} / \mathrm{kg}$, dissolved in $1 \mathrm{~N} \mathrm{HCl}$, neutralized with $1 \mathrm{~N} \mathrm{NaOH}$, diluted with saline), purchased from Sigma-Aldrich; Merck Millipore (Darmstadt, Germany) and fed for 4 weeks.

The rats were randomly divided into two groups ( $\mathrm{n}=6)$; in the control group, each rat was injected with a single dose of saline; in the PAH group, each rat was injected with a single dose of MCT (60 mg/kg). On day 28 , all rats were sacrificed.

Hemodynamic parameters. The rats were injected with $8 \%$ chloral hydrate following weighing $(1 \mathrm{ml} / 200 \mathrm{~g})$, and fixed on an autopsy table. Right ventricular systolic pressure (RVSP) was measured by insertion of a PE50 pipe through the jugular vein to the right ventricle; the RVSP was transferred into an electric signal and recorded using MedLab software (version 6.3; Nanjing Medease Science and Technology, Nanjing, China).

Measurement of right ventricular hypertrophy (RVH). All rats were sacrificed following measurement of pulmonary arterial pressure, and the hearts, lungs and pulmonary arteries were harvested. The blood was removed in cold PBS. The right ventricle (RV) was isolated from the left ventricle (LV) and septum (S), and these two components were weighted separately. RVH was determined as the ratio of RV weight to $(\mathrm{LV}+\mathrm{S})$ weight.

Histological analysis. A sample of lung tissue was fixed in prepared $4 \%$ paraformaldehyde for $24 \mathrm{~h}$, made into paraffinized sections (4- $\mu \mathrm{M}$ thick), and then stained with hematoxylin and eosin. A fluorescence microscope (Nikon Eclipse 80i; Nikon, Tokyo, Japan) was used to observe the pulmonary arteries in the stained sections.

Western blot analysis. The pulmonary arteries were cleared in cold PBS and frozen in liquid nitrogen prior to being stored at $-80^{\circ} \mathrm{C}$. The pulmonary artery tissues were homogenized in lysis buffer (radioimmunoprecipitation assay buffer, PMSF; 100:1) and then centrifuged at $13,800 \mathrm{x}$ g for $15 \mathrm{~min}$ at $4^{\circ} \mathrm{C}$. The protein concentrations were determined using a bicinchoninic protein assay kit, and $30 \mu \mathrm{g}$ were separated on a $10 \%$ SDS-PAGE gel, followed transferal onto a polyvinylidene difluoride membrane. The membrane was blocked in 5\% skim milk (5 g skim milk dissolved in $100 \mathrm{ml}$ Tris-buffered saline Tween solution) at room temperature for $1 \mathrm{~h}$. The membrane was incubated with the following antibodies: HMGR (cat. no. ab174830, 1:2,000), FDPS (cat. no. ab189874, 1:1,000), FNTA (cat. no. ab109738, 1:1,000), and FNTB (cat. no. ab109748, 1:1,000) (all from Abcam, Cambridge, UK), GGT-I (cat. no. sc18996, 1:200; Santa Cruz Biotechnology Co., Ltd., Dallas, TX, USA), phosphorylated (p)-eNOS (cat. no. 95719, 1:1,000), eNOS (cat. no. 9586, 1:1,000), and RhoA (cat. no. ARH04, 1:1,000) (all from CST Biological Reagents Company Limited, Shanghai, China), Rac1 (cat. no. ARC03, 1:1,000; BD Biosciences, Franklin Lakes, NJ, USA) at $4^{\circ} \mathrm{C}$ for $16 \mathrm{~h}$. The membranes were then incubated with the appropriate secondary antibody: Goat-anti-rabbit immunoglobulin G (IgG) (cat. no. 1268, 1:2,500), goat-anti-mouse IgG (cat. no. $1265,1: 2,500$ ), and rabbit-anti-goat IgG (cat. no. M1102, 1:2,500) (all from Biovision, Inc., Milpitas, CA, USA) for $2 \mathrm{~h}$ at room temperature. The target protein bands were visualized using chemiluminescence and quantified using ImageJ software (version 1.47; National Institutes of Health, Bethesda, MD, USA). GAPDH was used as an endogenous control (cat. no. 377R, 1:5,000; Biovison, Inc.). All antibodies were diluted in 5\% BSA (HuaBio, China).

NADPH oxidase activation assay. The activation of NADPH oxidase was detected using a Tissue NADPH Oxidase Activation Assay kit (Genmed Scientifics, Inc., Arlington, MA, USA). The pulmonary arteries were homogenized in lysis buffer and protein concentrations were determined using a BCA protein assay kit. According to the manufacturer's instructions, this was finally detected at $550 \mathrm{~nm}$ using a microplate reader (Thermo Fisher Scientific, Inc., Waltham, MA, USA.).

ROS kinase activation assay. A Tissue ROS Kinase Activation Assay kit (Genmed Scientifics, Inc.) was used to measure the level of ROS in the pulmonary artery. The pulmonary arteries were homogenized in lysis buffer and protein concentrations were determined using a BCA protein assay kit. The results were detected at $340 \mathrm{~nm}$ using a microplate reader (Thermo Fisher Scientific, Inc.). 

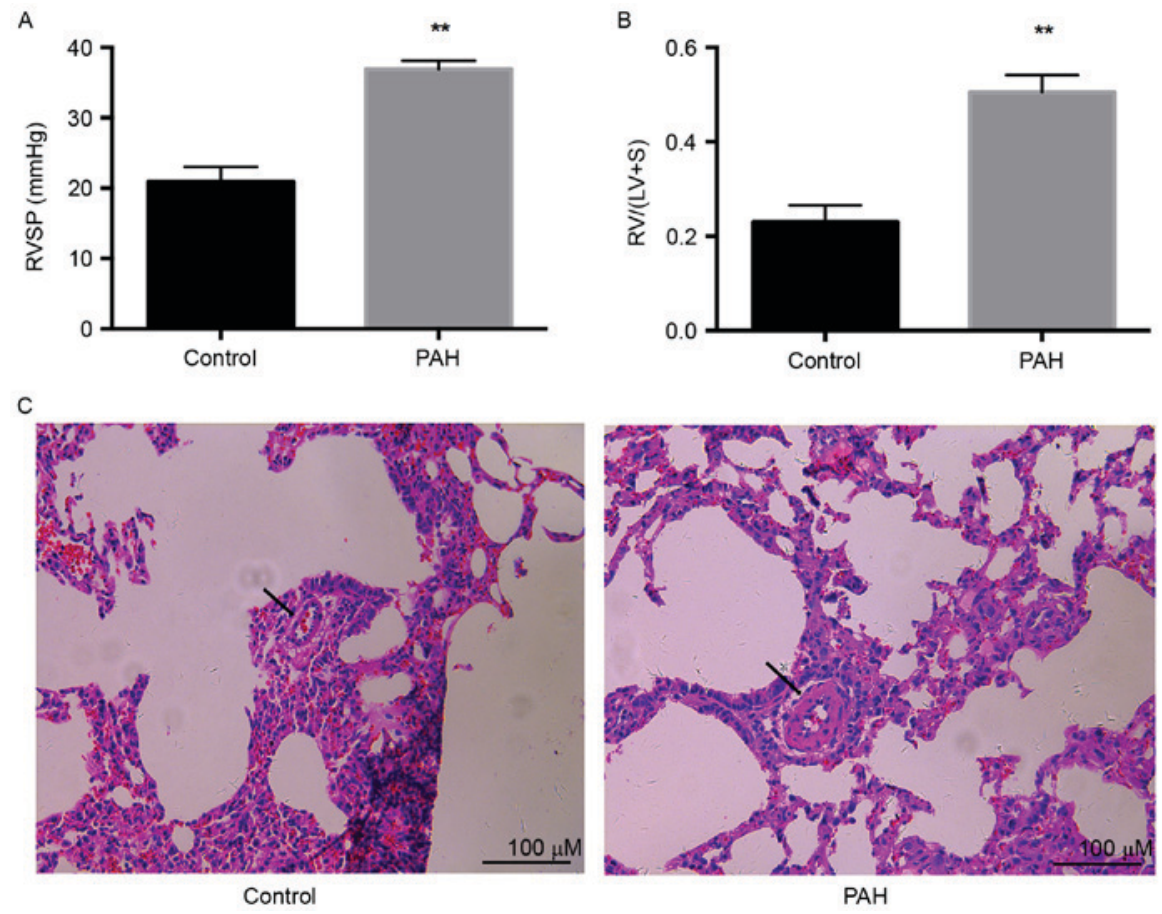

Figure 1. Development of PAH 4 weeks following a single injection of monocrotaline (60 mg/kg). (A) RVSP in control and PAH groups. Data are expressed as the mean \pm standard deviation. ${ }^{* *} \mathrm{P}<0.01$ compared with the control group. (B) $\mathrm{RV} /(\mathrm{LV}+\mathrm{S})$ in each group. (C) Hematoxylin and eosin staining of lung tissues. The arrows indicate pulmonary micro-arteries (original magnification, x200). RVSP, right ventricular systolic pressure; PAH, pulmonary arterial hypertension; RV, right ventricle; LV, left ventricle; S, septum.

Measurement of serum NO levels. The blood samples were collected and centrifuged at $13,8000 \mathrm{x} \mathrm{g}, 4^{\circ} \mathrm{C}$ for $15 \mathrm{~min}$. The serum was then removed into a new EP tube, and serum NO levels were determined using a Nitric Oxide Fluorometric Assay kit (Nanjing Jiancheng Bioengineering Institute, Nanjing, China) according to the manufacturer's instructions. The results were final detected using a microplate reader (Thermo Fisher Scientific, Inc.).

Statistical analysis. All values are expressed as the mean \pm standard error of the mean. Statistical significance was measured using one-way analysis of variance. Software used for analysis was GraphPad Prism (version 6.0c; GraphPad Software, Inc., La Jolla, CA, USA.). P $<0.05$ was considered to indicate a statistically significant difference.

\section{Results}

MCT-induced PAH. The PAH model was induced by injection of a single dose of MCT (60 mg/kg). After 4 weeks, RVSP was measured by insertion of a PE50 pipe through the jugular vein to the right ventricle. The pressures were transferred into electric signals and collected using MedLab software. The RVSP of the PAH group was significantly increased, compared with that in the control $(36.9 \pm 1.1$ vs. $20.9 \pm 1.1 ; \mathrm{P}<0.01)$ as exhibited in Fig. 1A. RVH, calculated as the ratio of RV weight to $(\mathrm{LV}+\mathrm{S})$ weight, was significantly increased by $\sim 2$-fold in the PAH group compared with that in the control $(0.50 \pm 0.02$ vs. $0.23 \pm 0.02$; $\mathrm{P}<0.01$ ), as exhibited in Fig. 1B. Pulmonary vascular remodeling was a notable characteristic of PAH. Significant thickening of micro-pulmonary arteries was demonstrated in the PAH group, compared with the control group (Fig. 1C). These results indicated that injection with a single dose of MCT $(60 \mathrm{mg} / \mathrm{kg})$ successfully induced PAH 4 weeks later.

Expression of key enzymes in the mevalonate pathway are altered in MCT-induced PAH. The present study aimed to determine whether the expression levels of key enzymes in the mevalonate pathway are altered in PAH. This involved comparing the expression levels of key enzymes in the pulmonary artery between the PAH and control groups.

HMGR is an initial enzyme in the mevalonate pathway, and the present study found no significant difference in its expression between the PAH and control group (Fig. 2A and B). A significant increase in the expression of FDPS was detected in the PAH group, compared with that in the control group $(\mathrm{P}<0.05)$, as exhibited in Fig. 2C. As FTase and GGTase-I catalyze the farnesylation for several small G-proteins, including the Ras and Rho family respectively $(18,19)$, the levels of FNTA and GGTase-I were significantly increased in the PAH group, compared with those in the control group $\mathrm{P}<0.01$ ), whereas no significant difference in FNTB was demonstrated (Fig. 2D-F). Overall, the expression levels of enzymes FDPS, FNTA and GGTase-I were elevated in the PAH rats.

Expression of small G-protein RhoA and Racl in MCT-induced $P A H$. Small G-proteins are important in regular specific cell function and gene expression (11), and evidence indicates that the prenylation and activation of small G-proteins are regulated by the enzymes GGTase-I and FTase (18). The expression levels of small G-protein RhoA and Rac1 in the present study were determined using western blot analysis (Fig. 3A). The level of RhoA was significantly elevated in the PAH group, compared with that in the control group (Fig. 3A 


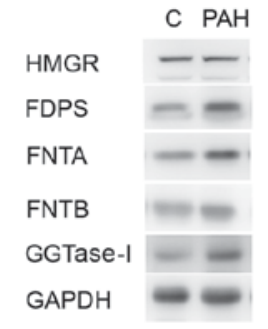

D

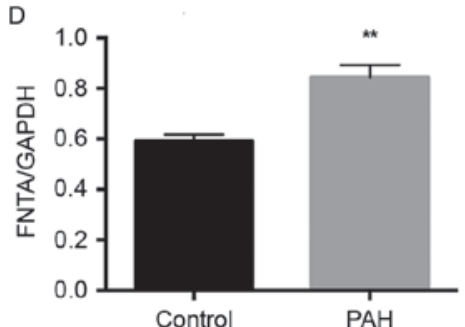

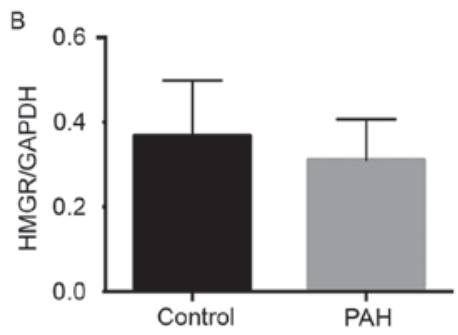

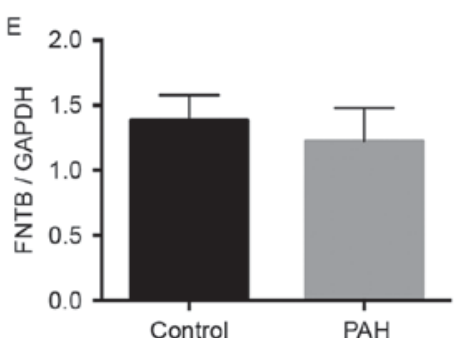

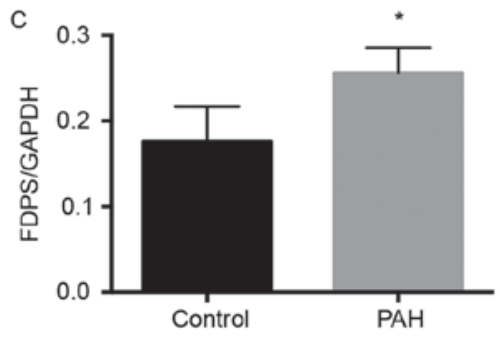

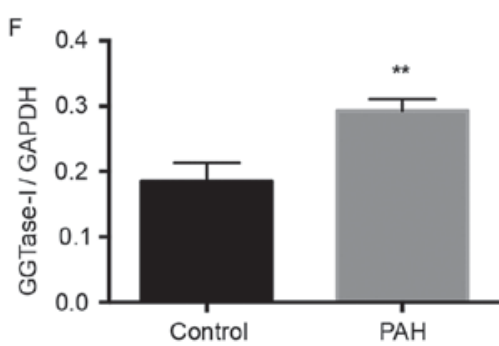

Figure 2. Expression levels of HMGR, FDPS, FNTA, FNTB and GGTase-I in the MCT-induced PAH rat pulmonary artery. The proteins were extracted from pulmonary arteries of each group. (A) Western blot analyses demonstrate the expression of HMGR, FDPS, FNTA, FNTB and GGTase-I in the control and MCT-induced PAH rats. GAPDH was used as the endogenous loading control. Graphs demonstrate the relative changes in (B) HMGR, (C) FDPS, (D) FNTA, (E) FNTB and (F) GGTase-I in the control and MCT groups. Data are expressed as the mean \pm standard deviation. " $\mathrm{P}<0.05$ and ${ }^{* *} \mathrm{P}<0.01$ compared with the control group. PAH, pulmonary arterial hypertension; MCT, monocrotaline; HMGR, 3-hydroxy-3-methylglutaryl-coenzyme A; FDPS, farnesyldiphosphate synthase; FNTA, farnesyltransferase $\alpha$; FNTB, farnesyltransferase $\beta$; GGTase-I, geranylgeranyltransferase type I.
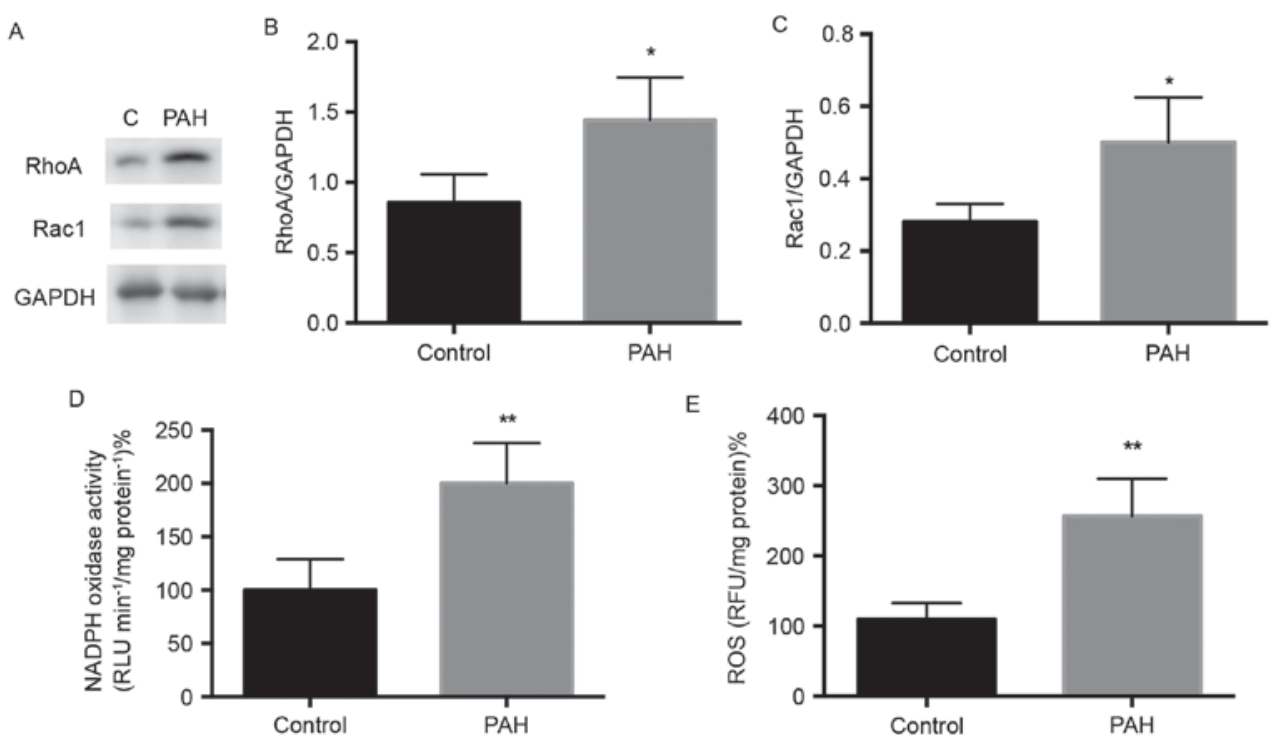

Figure 3. Oxidative stress and small-G protein expression in control and PAH rats. Proteins were extracted from the pulmonary artery of control and PAH rats. (A) Western blot analyses demonstrate the expression of RhoA and Rac1 in the control and PAH rats. GAPDH was used as an endogenous loading control. Graphs demonstrate the relative changes of (B) RhoA and (C) Racl in each group. (D) Changes in NADPH oxidase activity in each group were detected using a tissue NADPH oxidase activation assay kit. (E) ROS changes in each group were detected using a tissue ROS kinase activation assay kit. Data are expressed as the mean \pm standard deviation. ${ }^{*} \mathrm{P}<0.05$ and ${ }^{* *} \mathrm{P}<0.01$ compared with the control group. PAH, pulmonary arterial hypertension; ROS, reactive oxygen species; RhoA, Ras homolog family, member A; Rac1, Ras-related C3 botulinum toxin substrate 1; C, control.

and $\mathrm{B} ; \mathrm{P}<0.05)$, and Racl was also significantly increased in the $\mathrm{PAH}$ group (Fig. $3 \mathrm{~A}$ and $\mathrm{C} ; \mathrm{P}<0.05$ ). These results indicated that the expression of small G-proteins were increased in MCT-induced PAH rats.

NADPH activity is increased in MCT-induced PAH. NADPH oxidase is the downstream effector of small G-protein, and its activation depends on the Rac protein (20). The present study detected whether the expression of NADPH oxidase, the downstream effector, was altered in PAH. Following the treatment of protein lysates according to the manufacturer's protocol of the NADPH Oxidase Activation Assay kit, it was found that NADPH oxidase activity was increased by $\sim 2$-fold in the PAH group, compared with that in the control (Fig. 3D).

ROS kinase activity is increased in MCT-induced PAH. NADPH oxidase is a resource for generating ROS (21). The overexpression of ROS is involved in endothelial injury and vessel remodeling (8). The present study measured ROS kinase activity and found a significant increase in the PAH group, 
A

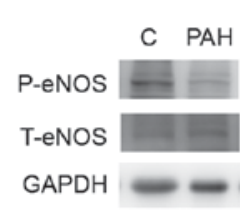

B

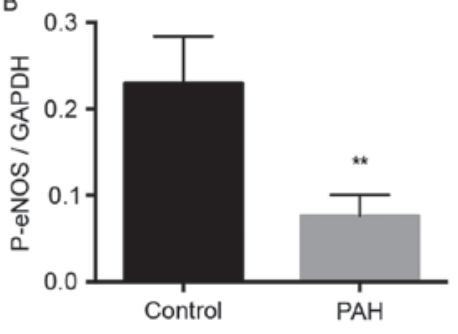

C

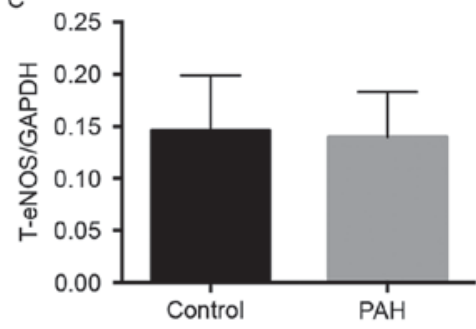

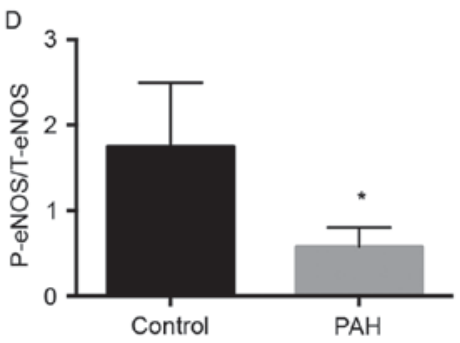

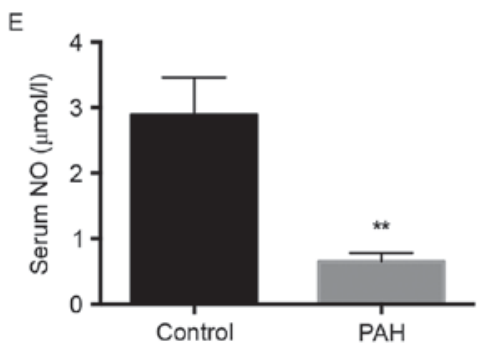

Figure 4. Alterations in eNOS activity and serum NO release in control and PAH rats. Proteins were extracted from the pulmonary artery of animals each group. (A) Western blot analyses demonstrate the expression of P-eNOS and T-eNOS in the control and PAH groups. GAPDH was used as an endogenous loading control. Graphs demonstrate the relative changes of (B) P-eNOS and (C) T-eNOS in each group. (D) Graph demonstrating the ratio of P-eNOS to T-eNOS. (E) Graph demonstrating the changes in serum NO release in the control and PAH groups. Data are expressed as the mean \pm standard deviation. ${ }^{*} \mathrm{P}<0.05$ and ${ }^{* *} \mathrm{P}<0.01$ compared with the control group. PAH, pulmonary arterial hypertension; NO, nitric oxide; eNOS, endothelial NO synthase; $\mathrm{P}$, phosphorylated; $\mathrm{T}$, total.

compared with that in the control (Fig. 3E; $\mathrm{P}<0.01$ ). This indicated that oxidative stress was elevated in the pulmonary artery of rats with MCT-induced PAH.

Activation of eNOS is decreased in MCT-induced PAH. The eNOS enzyme catalyzes the biosynthesis of NO, and NO in its molecular form has been reported to be important for the development of PAH (22). Therefore, the present study detected the expression of eNOS (Fig. 4A). The level of p-eNOS was significantly decreased in the PAH group, compared with that in the control group (Fig. 4B; $\mathrm{P}<0.01$ ), whereas no significant difference in total NOS was observed between the PAH and the control group (Fig. 4C). Therefore, the activity of eNOS, represented by the ratio of p-eNOS to total eNOS, was significantly decreased in the $\mathrm{PAH}$ group, compared with the control (Fig. 4C; $\mathrm{P}<0.05$ ).

Serum levels of NO are decreased in MCT-induced PAH. $\mathrm{NO}$ is an important molecule for cardiovascular health. It is released as an endothelium-derived relaxing factor (23). The present study detected the serum levels of NO using a Nitric Oxide Fluorometric Assay kit. The serum level of NO in the PAH group was significantly lower, compared with that in the control (Fig. 4D; $\mathrm{P}<0.01$ ). This may by due to the decreased activity of eNOS in the pulmonary artery of MCT-induced rats.

\section{Discussion}

PAH is a serious pulmonary vascular disease, which can lead to right heart failure following qualitative changes in the artery and lumen, blood flow and pressure, and cardiac muscle $(2,24)$. In the present study, changes in the expression of key enzymes of the mevalonate pathway were detected in PAH rats. The MCT-induced PAH model is an established method generally used in rat experiments (25). In the present study, initial measurements of RVSP revealed a significantly increase in the PAH group 4 weeks following MCT injection. This result was accompanied by a high ratio of $\mathrm{RV} /(\mathrm{LV}+\mathrm{S})$ in the $\mathrm{PAH}$ group. These results indicated that the PAH model and right ventricular remodeling had been successfully established.

In the present study, it was demonstrated that the expression of key enzymes in the mevalonate pathway, including FDPS, FNTA and GGTase-I, were significantly increased in the pulmonary artery of MCT-induced PAH rats. The expression of small G-protein Racl and RhoA were also increased, which was consistent with the results of GGTase-I and FTase. Small G-proteins downstream effectors, including NADPH and ROS, were also examined; significant increases in ROS and NADPH oxidase activity were demonstrated, whereas the protein expression of eNOS and release of serum NO decreased.

HMGR is the initial enzyme in cholesterol synthesis. The therapeutic effect of statins on pulmonary hypertension is controversial in clinical trails. In the present study, no significant change was demonstrated in HMGR in the PAH rats, consistent with a previous meta-analysis, which reported that HMGR inhibitor statins had no benefit in patients with PAH (26). Isoprenoid is the intermediate of the mevalonate pathway, and is important for diverse cellular functions (12). The three known end-products of isoprenoid are cholesterol, dolichol and the polyisoprene side chain of ubiquinone (27). Steroidal and non-steroidal isoprenoids partially regulate the expression of enzymes in the mevalonate pathway (28). FDPS is an important enzyme in the mevalonate pathway, which catalyzes the synthesis of FPP and GPP from the substrate mevalonate (28). In the present study, an increase in the enzyme FDPS was found in the pulmonary artery tissue of the PAH rats. This increased expression of FDPS may be responsible for pulmonary vessel remodeling and right ventricular hypertrophy. Therefore, FDPS 
inhibitors are being investigated as a treatment option for certain diseases in which FDPS is overexpressed. A previous study demonstrated inhibiting FDPS by N6-isopentenyladenosine, an adenosine and isoprenoid derivative, was demonstrated to inhibit the proliferation of tumor cells (29). In addition, bisphosphonates are widely used FDPS inhibitor in the treatment of osteoporosis (30). The chronic inhibition of FDPS can also attenuate cardiac hypertrophy and fibrosis (31), although the specific signaling mechanism remains to be elucidated. It was hypothesized that the abnormal expression of FDPS may be a potential therapeutic target for the treatment of PAH.

The higher level of FDPS induced the accumulation of downstream products, including GPP and FPP. As an intermediate product, FPP is an important precursor in the synthesis of sterols, dolichols and ubiquinones (32). It is well known that the activation of proteins, including Ras and Rab, require farnesylation by FTase with FPP as substrate in the transmembrane, which responds to cellular signaling. Therefore, Ras can activate genes involved in cell growth, proliferation, and differentiation, and lead to abnormal vessel growth and remodeling (33). The present study demonstrated that the expression levels of FTase and GGTase-I were increased in the PAH group. The enzyme GGTase-I catalyzes the gernalygernalation of proteins, including the Rho family and Rac. The elevated expression level of GGTase-I has been reported in several human diseases, including renal fibrosis, spontaneous hypertension and cancer (34). Previous studies have attempted to use GGTase-I inhibitor to treat diseases, including renal fibrosis and cancer, and have achieved promising results (35). In order to localize in correct subcellular membranes, small G-proteins of the Rac and RhoA family require post-translational prenylation by FTase and GGTase-I, and then transducing signals to downstream effectors (36). Unlike certain cardiovascular diseases, including pressure overload-induced cardiac hypertrophy and spontaneous hypertension, in which the expression of FNTB is elevated (37), the present study found no significant change in the expression of FNTB in the PAH model. This may be due to differences in tissue expression and disease models, indicating that FNTA is more important in the development of PAH than FNTB.

The Rho family, including Rac and Rho, can regulate the function of the cytoskeleton (38). Rho proteins are involved in the activation of extracellular signal-regulated kinase (ERK) in response to angiotensin II or stretch-induced hypertrophy (39). Certain studies have demonstrated that Rac can provoke thec-Jun N-terminal kinase/p38 subgroup of nuclear mitogenactivated protein kinases (MAPKs) (40). Activated Rac can stimulate the activity of p21-activated kinase (PAK) (41), and activated PAK can affect the activation of ERK via the phosphorylation of the serine/threonine protein kinase Raf (42). The transcription of nuclear genes associated with cell proliferation and growth can be regulated following the activation of Raf, MAPK and ERK proteins $(43,44)$.

NADPH oxidase is a membrane-bound enzyme complex. Several studies have shown that the activation of NADPH oxidase depends on the Rac protein and two other cytosolic proteins, p47phox and p67phox (10). Rac-GDP is converted into Rac-GTP and translocated to the correct membrane, and NADPH oxidase is activated (45). The present study measured the activity of NADPH oxidase and found it was markedly increased in the PAH rats, consistent with the increase of Rac1. This result was supported by a previous study, which demonstrated the same increased activity of NADPH oxidase in an MCT-induced PAH model (46).

NADPH oxidase is a source of superoxides, and superoxides undergo further reactions to generate ROS (47). According to the present study, the PAH rats generated more NADPH-derived ROS, compared with the control rats, which was consistent with previous studies $(46,48)$. ROS is an essential regulator of normal cell physiology. The overproduction of ROS is associated with cardiovascular disorders, metabolic dysfunction and other diseases $(47,49)$, therefore, it was hypothesized that generated ROS contributes to the development of PAH. Accumulated ROS may function through reacting with cellular components, affecting the function of endothelial cells, inducing the proliferation of smooth muscle cells, and vascular remodeling $(50,51)$.

In several heart diseases, $\mathrm{NO}$ is an important molecule in vasodilation. The present study found decreased activity of eNOS and serum levels of NO in the PAH group. Although the bio-activity of eNOS was weaker in PAH, no differences in the expression of eNOS were demonstrated between PAH and the control group. This contradicted the results of a previous study, which reported that the expression level of eNOS decreased in the PAH model (52). However, previous studies commonly used endothelial cells, including human umbilical vein endothelial cells, whereas the experiments in the present study used whole pulmonary artery tissue. This may be the reason for the difference in results. Reductions in NO have not been observed in previous studies (53). The superoxide-like ROS can react with NO to reduce NO bioavailability (9), and the imbalance of ROS-NO levels may lead to endothelial dysfunction, consequently stimulating the development of PAH.

The present study detected changes in the expression of key enzymes of the mevalonate pathway in an MCT-induced PAH model, which may serve as drug targets for PAH treatment. The inhibitors of certain enzymes, including FDPS and GGTase-I, have been used in the treatment of certain human diseases. However, whether treatment with the inhibitors of altered enzymes in PAH can attenuate persistent pulmonary artery remodeling and high pressure remains to be elucidated, for which further investigations are required.

\section{Acknowledgements}

This study was financially supported by the National Natural Sciences Foundation of China (grant no. 81400277) and the Natural Sciences Foundation of Zhejiang Province (grant no. LY17H020006). The abstract was presented at the 15 th meeting of China Interventional Therapeutics March 30 - April 2, 2017 in Beijing, China (abstract no. AS-0341).

\section{References}

1. Hoeper MM, Bogaard HJ, Condliffe R, Frantz R, Khanna D, Kurzyna M, Langleben D, Manes A, Satoh T, Torres F, et al: Definitions and diagnosis of pulmonary hypertension. J Am Coll Cardiol 62 (Suppl 25): D42-D50, 2013.

2. Malenfant S, Margaillan G, Loehr JE: Bonnet Sb and Provencher S: The emergence of new therapeutic targets in pulmonary arterial hypertension: From now to the near future. Expert Rev Respir Med 7: 43-55, 2013. 
3. Houtchens J, Martin D and Klinger JR: Diagnosis and management of pulmonary arterial hypertension. Pulmonary Med 2011: $1-13,2011$.

4. Mam V, Tanbe AF, Vitali SH, Arons E, Christou HA and Khalil RA Impaired vasoconstriction and nitric oxide-mediated relaxation in pulmonary arteries of hypoxia- and monocrotaline-induced pulmonary hypertensive rats. J Pharmacol Exp Ther 332: 455-462, 2010.

5. Giaid A and Saleh D: Reduced expression of endothelial nitric oxide synthase in the lungs of patients with pulmonary hypertension. N Engl J Med 333: 214-221, 1995.

6. Goldstein JL and Brown MS: Regulation of the mevalonate pathway. Nature 343: 425-430, 1990.

7. Klinger JR, Abman SH and Gladwin MT: Nitric oxide deficiency and endothelial dysfunction in pulmonary arterial hypertension. Am J Respir Crit Care Med 188: 639-646, 2013.

8. Cai H and Harrison DG: Endothelial dysfunction in cardiovascular diseases: The role of oxidant stress. Circ Res 87: 840-844, 2000.

9. Dorfmüller P, Chaumais MC, Giannakouli M, Durand-Gasselin I, Raymond N, Fadel E, Mercier O, Charlotte F, Montani D, Simonneau G, et al: Increased oxidative stress and severe arterial remodeling induced by permanent high-flow challenge in experimental pulmonary hypertension. Respir Res 12: 119-130, 2011.

10. Rinckel LA, Faris SL, Hitt ND and Kleinberg ME: Racl disrupts p67phox:p40phox binding: A novel role for rac in NADPH oxidase activation. Biochem Biophys Res Commun 263: 118-122, 1999.

11. Takai Y, Sasaki T and Matozaki T: Small GTP-binding proteins Physiol Rev 81: 154-185, 2001.

12. Allal C, Favre G, Couderc B, Salicio S, Sixou S, Hamilton AD, Sebti AM, Lajoie-Mazenc I and Pradines A: RhoA prenylation is required for promotion of cell growth and transformation and cytoskeleton organization but not for induction of serum response element transcription. J Biol Chem 275: 31001-31008, 2000.

13. Singh RP, Kumar R and Kapur N: Molecular regulation of cholesterol biosynthesis: Implications in carcinogenesis. J Environ Pathol Toxicol Oncol 22: 75-92, 2003.

14. Kinlay S: Potential vascular benefits of statins. Am J Med 118 (Suppl 12A): S62-S67, 2005.

15. Koh KK: Effects of statins on vascular wall: Vasomotor function, inflammation and plaque stability. Cardiovasc Res 47: 648-657, 2000.

16. Murata T, Kinoshita K, Hori M, Kuwahara M, Tsubone H, Karaki $\mathrm{H}$ and Ozaki $\mathrm{H}$ : Statin protects endothelial nitric oxide synthase activity in hypoxia-induced pulmonary hypertension. Arterioscler Thromb Vasc Biol 25: 2335-2342, 2005.

17. Guerard P, Rakotoniaina Z, Goirand Fo, Rochette L, Dumas M, Lirussi $\mathrm{F}$ and Bardou M: The HMG-CoA reductase inhibitor, pravastatin, prevents the development of monocrotaline-induced pulmonary hypertension in the rat through reduction of endothelial cell apoptosis and overexpression of eNOS. Naunyn Schmiedebergs Arch Pharmacol 373: 401-414, 2006.

18. Zhang FL and Casey PJ: Protein prenylation: Molecular mechanisms and functional consequences. Annu Rev Biochem 65 241-269, 1996

19. Moores SL, Schaber MD, Mosser SD, Rands E, O'Hara MB Garsky VM, Marshall MS, Pompliano DL and Gibbs JB: Sequence dependence of protein isoprenylation. J Biol Chem 266 14603-14610, 1991.

20. Lőrincz ÁM, Szarvas G, Smith SM and Ligeti E: Role of Rac GTPase activating proteins in regulation of NADPH oxidase in human neutrophils. Free Radic Biol Med 68: 65-71, 2014.

21. Seno T, Inoue N, Gao D, Okuda M, Sumi Y, Matsui K, Yamada S, Hirata KI, Kawashima S, Tawa R, et al: Involvement of NADH/ NADPH oxidase in human platelet ROS production. Thromb Res 103: 399-409, 2001.

22. Machado RF, Londhe Nerkar MV, Dweik RA, Hammel J, Janocha A, Pyle J, Laskowski D, Jennings C, Arroliga AC and Erzurum SC: Nitric oxide and pulmonary arterial pressures in pulmonary hypertension. Free Radic Biol Med 37: 1010-1017, 2004.

23. Crosswhite $\mathrm{P}$ and Sun Z: Mol mechanisms of pulmonary arterial remodeling. Mol Med 20: 191-201, 2014.

24. Yerly P, Prella M and Aubert JD: Current management of pulmonary arterial hypertension. Swiss Med Wkly 146: w14305, 2016.

25. Guzowski DE and Salgado ED: Changes in main pulmonary artery of rats with monocrotaline-induced pulmonary hypertension. Arch Pathol Lab Med 111: 741-745, 1987.

26. Anand V, Garg S, Duval S and Thenappan T: A systematic review and meta-analysis of trials using statins in pulmonary arterial hypertension. Pulm Circ 6: 295-301, 2016.
27. Faust JR, Goldstein JL and Brown MS: Synthesis of ubiquinone and cholesterol in human fibroblasts: Regulation of a branched pathway. Arch Biochem Bioph 192: 86-99, 1979.

28. Brown MS and Goldstein JL: Multivalent feedback regulation of HMG CoA reductase, a control mechanism coordinating isoprenoid synthesis and cell growth. J Lipid Res 21: 505-517, 1980.

29. Laezza C, Notarnicola M, Caruso MG, Messa C, Macchia M, Bertini S, Minutolo F, Portella G, Fiorentino L, Stingo S and Bifulco M: N6-isopentenyladenosine arrests tumor cell proliferation by inhibiting farnesyl diphosphate synthase and protein prenylation. FASEB J 20: 412-418, 2006.

30. Park SB, Park SH, Kang YK and Chung CK: The time-dependent effect of ibandronate on bone graft remodeling in an ovariectomized rat spinal arthrodesis model. Spine J 14: 1748-1757, 2014.

31. Li L, Chen GP, Yang Y, Ye Y, Yao L and Hu SJ: Chronic inhibition of farnesyl pyrophosphate synthase attenuates cardiac hypertrophy and fibrosis in spontaneously hypertensive rats. Biochem Pharmacol 79: 399-406, 2010.

32. Sun S and McKenna CE: Farnesyl pyrophosphate synthase modulators: A patent review (2006-2010). Expert Opin Ther Pat 21: 1433-1451, 2011.

33. Bajaj A, Zheng Q, Adam A, Vincent P and Pumiglia K: Activation of endothelial ras signaling bypasses senescence and causes abnormal vascular morphogenesis. Cancer Res 70: 3803-3812, 2010.

34. Wiemer AJ, Wiemer DF and Hohl RJ: Geranylgeranyl diphosphate synthase: An emerging therapeutic target. Clin Pharmacol Ther 90: 804-812, 2011.

35. Philips MR and Cox AD: Geranylgeranyltransferase I as a target for anti-cancer drugs. J Clin Invest 117: 1223-1225, 2007.

36. Dunford JE, Rogers MJ, Ebetino FH, Phipps RJ and Coxon FP: Inhibition of protein prenylation by bisphosphonates causes sustained activation of Rac, Cdc42, and Rho GTPases. J Bone Miner Res 21: 684-694, 2006.

37. Han J, Jiang DM, Du CQ and Hu SJ: Alteration of enzyme expressions in mevalonate pathway: Possible role for cardiovascular remodeling in spontaneously Hypertensive Rats. Circ J 75: 1409-1417, 2011.

38. Hall A: Small GTP-binding proteins and the regulation of the actin cytoskeleton. Ann Rev Cell Biol 10: 31-54, 1994.

39. Aikawa R, Komuro I, Yanazaki T, Zou Y, Kudoh S, Zhu W, Kadowaki $\mathrm{T}$ and Yazaki Y: Rho family small $\mathrm{G}$ proteins play critical roles in mechanical stress-induced hypertrophic responses in cardiac myocytes. Circ Res 84: 485-466, 1999.

40. Bagrodia S, Dérijard B, Davis RJ and Cerione RA: Cdc42 and PAK-mediated Signaling Leads to Jun kinase and p38 mitogen-activated protein kinase activation. J Biol Chem 270: 27995-27998, 1995.

41. Bagrodia $\mathrm{S}$ and Cerione RA: PAK to the future. Trends Cell Biol 9: 350-355, 1999.

42. King AJ, Sun H, Diaz B, Barnard D, Miao W, Bagrodia S and Marshall MS: The protein kinase Pak3 positively regulates Raf-1 activity through phosphorylation of serine 338. Nature 396: 180-183, 1998.

43. Klemke RL, Cai S, Giannini AL, Gallagher PJ, de Lanerolle P and Cheresh DA: Regulation of cell motility by mitogen-activated protein kinase. J Cell Biol 137: 481-492, 1997.

44. Hunter JJ, Tanaka N, Rockman HA, Ross J and Chien KR: Ventricular expression of a MLC-2v-ras fusion gene induces cardiac hypertrophy and selective diastolic dysfunction in transgenic mice. J Biol Chem 270: 23173-23178, 1995

45. Norton CE, Broughton BR, Jernigan NL, Walker BR and Resta TC: Enhanced depolarization-induced pulmonary vasoconstriction following chronic hypoxia requires EGFR-dependent activation of NAD $(\mathrm{P}) \mathrm{H}$ oxidase 2. Antioxid Redox Signal 18: 1777-88, 2013.

46. Kameshima S, Kazama K, Okada $\mathrm{M}$ and Yamawaki $\mathrm{H}$ Eukaryotic elongation factor 2 kinase mediates monocrotaline-induced pulmonary arterial hypertension via reactive oxygen species-dependent vascular remodeling. Am J Physiol Heart Circ Physiol 308: H1298-H1305, 2015.

47. Manea A: NADPH oxidase-derived reactive oxygen species: Involvement in vascular physiology and pathology. Cell Tissue Res 342: 325-339, 2010

48. Sutendra G, Dromparis P, Bonnet S, Haromy A, McMurtry MS, Bleackley RC and Michelakis ED: Pyruvate dehydrogenase inhibition by the inflammatory cytokine TNFalpha contributes to the pathogenesis of pulmonary arterial hypertension. J Mol Med (Berl) 89: 771-783, 2011 
49. Aziz SM, Toborek M, Hennig B, Mattson MP, Guo H and Lipke DW: Oxidative stress mediates monocrotaline-induced alterations in tenascin expression in pulmonary artery endothelial cells. Int J Biochem Cell Biol 29: 775-787, 1997.

50. Chen MJ, Chiang LY and Lai YL: Reactive oxygen species and substance $\mathrm{P}$ in monocrotaline-induced pulmonary hypertension. Toxicol Appl Pharmacol 171: 165-73, 2001.

51. Herbert JM, Bono F and Savi P: The mitogenic effec of $\mathrm{H}_{2} \mathrm{O}_{2}$ for vascular smooth muxcle cells is mediated by an increase of the affinity of basid fibroblast growth factor for its receptor. FEBS Lett 395: 43-47, 1996
52. Xu W, Kaneko FT, Zheng S, Comhair SA, Janocha AJ, Goggans T, Thunnissen FB, Farver C, Hazen SL, Jennings C, et al: Increased arginase II and decreased NO synthesis in endothelial cells of patients with pulmonary arterial hypertension. FASEB J 18: 1746-1748, 2004

53. Sahara M, Sata M, Morita T, Hirata Y and Nagai R: Nicorandil attenuates monocrotaline-induced vascular endothelial damage and pulmonary arterial hypertension. PLoS One 7: e33367, 2012. 